\title{
Damage Control Surgery Saves Patient with Gastric Lymphoma from Radical Gastrectomy
}

\author{
Gang Chen ${ }^{1}$, Min-min Zhang ${ }^{2}$, Jun-min Zhu' ${ }^{1}$, jie $\mathrm{Mao}^{1}$ and Yu-min $\mathrm{Li}^{1}$ \\ ${ }^{1}$ Department of General Surgery, Second Hospital of Lanzhou University, Lanzhou, Gansu, China \\ ${ }^{2}$ School of Clinical Medicine, Gansu University of Chinese Medicine, Lanzhou, Gansu, China
}

\begin{abstract}
The concept of damage control surgery was first introduced in the 1990s. It has great significance in the treatment for critically ill patients, which not only greatly improves survival rate, but also helps doctors avoiding misdiagnosis and mistreatment. Herein, we present a case of gastric perforation caused by neoplasm with critical condition of the patient. According to the concept of damage control surgery, the patient was subjected to perforation repair and tumor biopsy instead of conventional radical gastrectomy. Then, diffuse large B cell lymphoma was diagnosed on pathologic examination. After surgery, the patient received R-CHOP chemotherapy according to the clinical guidelines and is alive till now. Our experience might be helpful for understanding the value of damage control surgery in avoiding misdiagnosis and mistreatment for critical emergency patients.
\end{abstract}

Key Words: Lymphoma, Stomach, Perforation, Damage control surgery.

How to cite this article: Chen G, Zhang MM, Zhu JM, Mao J, Li YM. Damage Control Surgery Saves Patient with Gastric Lymphoma from Radical Gastrectomy. J Coll Physicians Surg Pak 2021; 31(08):978-981.

\section{INTRODUCTION}

In the early 1980s, Stone et al. proposed the concept of damage control surgery as treatment strategy and timing of surgery for severe trauma patients. ${ }^{1}$ In 1993, Rotondo et al. put forward the concept of damage control surgery. ${ }^{2}$ The essence of this concept is staged surgery protocol, by giving simple but effective treatment before radical surgery to correct the physiological imbalance and internal milieu disorder. The concept is not only useful for complex trauma and war wounds, but also has a significant role in non-traumatic critically ill patients. ${ }^{3,4}$

In this case, we would like to provide evidence that damage control surgery can help doctors to avoid misdiagnosis and mistreatment.

\section{CASE REPORT}

A 60-year man was admitted to hospital for sudden onset upper abdominal pain with progressive worsening and accompanied by fever for 6 hours. For the past year, he has had intermittent upper abdominal pain, which was relieved by taking omeprazole orally.

Correspondence to: Dr. Yu-min Li, Department of General Surgery, Second Hospital of Lanzhou University, Lanzhou 730030, Gansu, China

E-mail: lilz2019@163.com

Received: October 30, 2019; Revised: January 25, 2020; Accepted: February 13, 2020

DOI: https://doi.org/10.29271/jcpsp.2021.08.978
Physical examination results were as follows: passive flexion position, temperature $38.6^{\circ} \mathrm{C}$, pulse 125 beats $/ \mathrm{m}$, respiratory rate 30 breaths $/ \mathrm{m}$, blood pressure $130 / 80 \mathrm{mmHg}$, board-like abdomen, abdominal muscle tension, tenderness and rebound tenderness, and no audible bowel sounds. Laboratory tests showed abnormal chemical indicators: white blood cell count (WBC) $10.40 \times 10^{9} / \mathrm{L}$, platelets $78 \times 10^{9} / \mathrm{L}$, prothrombin time (PT) $12.2 \mathrm{~s}$ (Table 1 ), D-dimer $1.01 \mathrm{mg} / \mathrm{L}$, glucose (GLU) $9.66 \mathrm{mmol} / \mathrm{L}$, total bilirubin (TBIL) 34.4 $\mu \mathrm{mol} / \mathrm{L}$, direct bilirubin (DBIL) $15.7 \mu \mathrm{mol} / \mathrm{L}$, and indirect bilirubin (IBIL) $18.7 \mu \mathrm{mol} / \mathrm{L}$. Chest radiograph and abdominal computed tomography (CT) showed free gas in the abdominal cavity and a mass in the anterior wall of the gastric antrum (Figure $1 A, B, C$ ). The provisional diagnoses made were: acute upper gastrointestinal perforation acute diffuse peritonitis, and suspected gastric cancer.

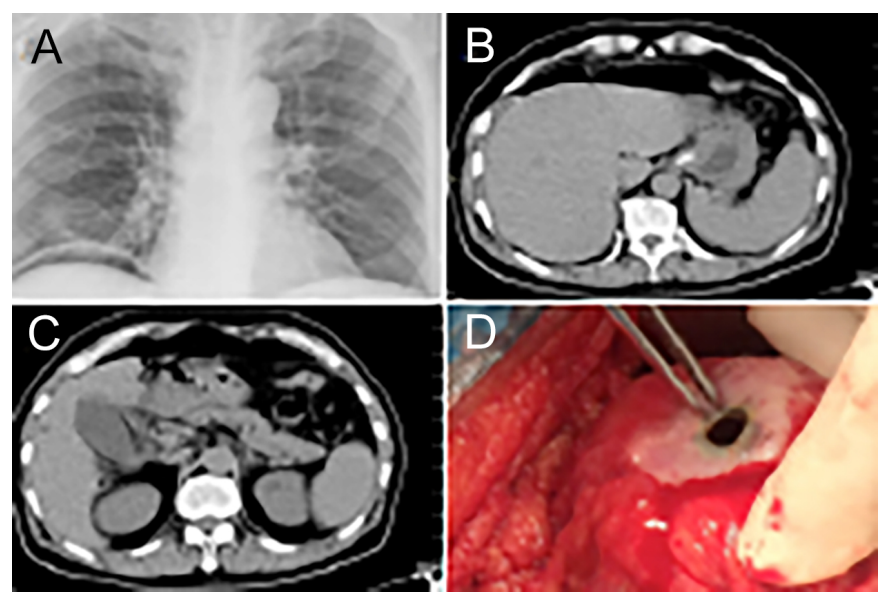

Figure 1: Preoperative examinations and intraoperative images. Chest radiograph showed free gas in the abdominal cavity (A); Abdominal CT scan showed free gas and peritoneal effusion (B, C); Antrum anterior wall tumor was found with perforation(D). 
Table I: Routine blood examinations. 2017.10.13, WC $10.4 \times 10^{9} / \mathrm{L}$, NE\% $88 \%$, PLT $78 \times 10^{9} / \mathrm{L}$. It is a sign of a serious infection, they gradually returned to normal after the operation. In the course of postoperative chemotherapy, WC was significantly reduced and stabilised at the normal level after the lymphoma was cured (2018.5.4-2019.1.16).

\begin{tabular}{|c|c|c|c|c|c|c|c|c|c|c|c|c|c|}
\hline Date & $\begin{array}{l}\mathrm{NE} \\
(\%)\end{array}$ & LY (\%) & $\begin{array}{l}\text { MO } \\
(\%)\end{array}$ & $\begin{array}{l}\text { EO } \\
(\%)\end{array}$ & $\begin{array}{l}\text { BA } \\
(\%)\end{array}$ & $\begin{array}{c}\text { WBC } \\
\left(\times 10^{9} / L\right)\end{array}$ & $\begin{array}{c}\text { NE } \\
\left(\times 10^{9} / L\right)\end{array}$ & $\begin{array}{c}\text { LY } \\
\left(\times 10^{9} / \mathrm{L}\right) \\
\end{array}$ & $\begin{array}{c}\text { MO } \\
\left(\times 10^{9} / L\right)\end{array}$ & $\begin{array}{c}\text { EO } \\
\left(\times 10^{9} / L\right)\end{array}$ & $\begin{array}{c}\text { BA } \\
\left(\times 10^{9} / \mathrm{L}\right) \\
\end{array}$ & $\begin{array}{c}\text { RBC } \\
\left(\times 10^{12} / \mathrm{L}\right)\end{array}$ & $\begin{array}{c}\text { PLT } \\
\left(\times 10^{9} / \mathrm{L}\right)\end{array}$ \\
\hline 2017.10 .13 & 0.88 & 0.05 & 0.07 & 0 & 0 & 10.4 & 9.17 & 0.54 & 0.68 & 0 & 0.01 & 5.83 & 78 \\
\hline 2017.10 .14 & 0.84 & 0.1 & 0.06 & 0 & 0 & 9.4 & 7.88 & 0.9 & 0.6 & 0.01 & 0.01 & 4.5 & 56 \\
\hline 2017.10 .15 & 0.85 & 0.08 & 0.06 & 0 & 0 & 8.1 & 6.93 & 0.68 & 0.49 & 0.06 & 0.01 & 4.07 & 64 \\
\hline 2017.10 .16 & 0.8 & 0.12 & 0.07 & 0.01 & 0 & 5.4 & 4.4 & 0.66 & 0.37 & 0.05 & 0.01 & 3.78 & 87 \\
\hline 2017.10 .18 & 0.72 & 0.19 & 0.07 & 0.01 & 0.01 & 6.3 & 4.51 & 1.21 & 0.46 & 0.06 & 0.03 & 3.48 & 128 \\
\hline 2017.10 .27 & 0.73 & 0.16 & 0.09 & 0 & 0 & 6.69 & 4.89 & 1.1 & 0.62 & 0.03 & 0.01 & 3.88 & 252 \\
\hline 2017.11 .22 & 0.39 & 0.51 & 0.08 & 0.02 & 0 & 3.2 & 1.26 & 1.63 & 0.27 & 0.07 & 0.01 & 4.26 & 104 \\
\hline 2017.12 .13 & 0.49 & 0.42 & 0.09 & 0 & 0 & 2.8 & 1.36 & 1.17 & 0.26 & 0 & 0 & 4.53 & 85 \\
\hline 2018.01 .18 & 0.47 & 0.38 & 0.15 & 0 & 0 & 2.3 & 1.09 & 0.88 & 0.33 & 0 & 0 & 4.04 & 84 \\
\hline 2018.02 .27 & 0.72 & 0.16 & 0.11 & 0 & 0 & 3.59 & 2.61 & 0.58 & 0.4 & 0 & 0 & 4.41 & 88 \\
\hline 2018.03 .26 & 0.66 & 0.18 & 0.16 & 0 & 0 & 2.83 & 1.88 & 0.5 & 0.44 & 0 & 0.01 & 3.63 & 169 \\
\hline 2018.04 .09 & 0.76 & 0.13 & 0.1 & 0.01 & 0.01 & 1.87 & 1.41 & 0.24 & 0.19 & 0.01 & 0.01 & 2.8 & 71 \\
\hline 2018.05 .04 & 0.76 & 0.13 & 0.11 & 0 & 0 & 4.01 & 3.04 & 0.53 & 0.43 & 0 & 0.01 & 3.49 & 116 \\
\hline 2018.12 .04 & 0.64 & 0.27 & 0.07 & 0.01 & 0 & 4.1 & 2.6 & 1.11 & 0.3 & 0.03 & 0.01 & 5.54 & 94 \\
\hline
\end{tabular}

\section{A}

A $\quad$ C

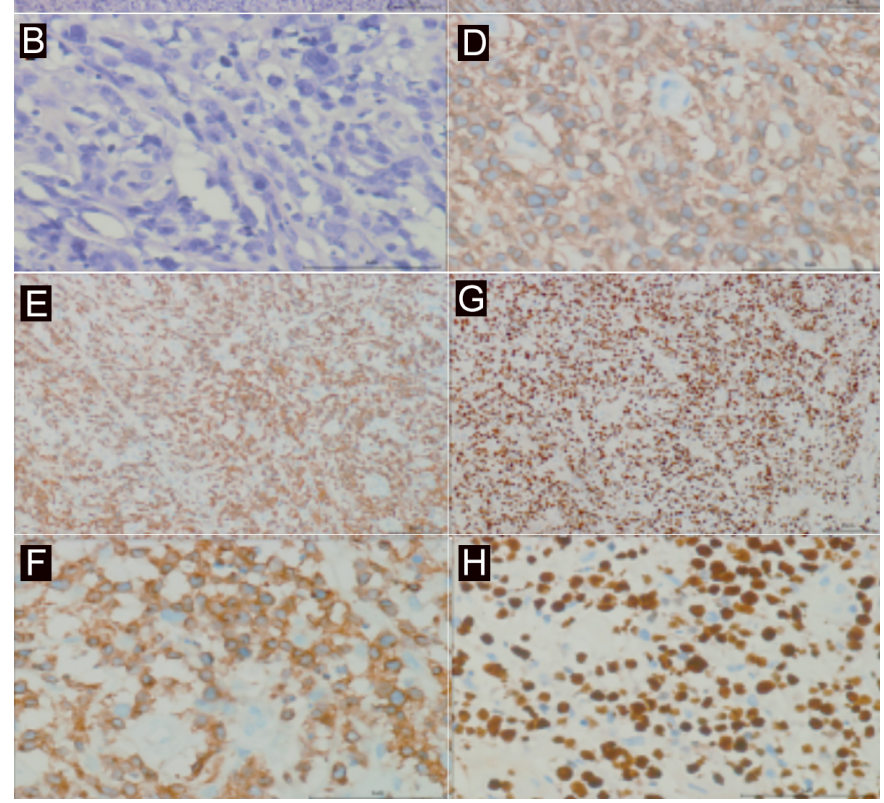

Figure 2: Pathological examinations. Extensive lymphocyte infiltration in HE staining ( $A 10 x, B 40 x$ ), immunohistochemical examination showed CD20 (+), C 10x, D 40x; CD79a (+), E 10x, F 40x; Ki67(+, 80\%).

An emergency laparotomy was performed. Results of intraoperative exploration were as follows: (1) removal of $1100 \mathrm{ml}$ greyish white pus in the abdominal cavity, (2) two small hemangiomas in the liver, about $2 \times 4 \mathrm{~cm}$ and $3 \times 3 \mathrm{~cm}$ in size, (3) highly edematous and thickened greater omentum, gastrointestinal tract and mesentery, and (4) a tumor in the anterior wall of the gastric antrum, $5 \mathrm{~cm}$ in diameter, with a perforation of $1.5 \mathrm{~cm}$ in diameter (Figure 1D). Therefore, the intraoperative diagnosis was gastric neoplasm (pathology undetermined) with perforation, diffuse peritonitis, abdominal infection, and hepatic hemangioma. Meanwhile, the patient's condition was cumulatively assessed as follows: (1) affirmative gastric tumor with perforation, but the pathology result was unclear, (2) severe abdominal organ edema, massive fluid loss, unstable hemodynamics, diabetes, and viral hepatitis, which disabled the patient for radical gastrectomy.

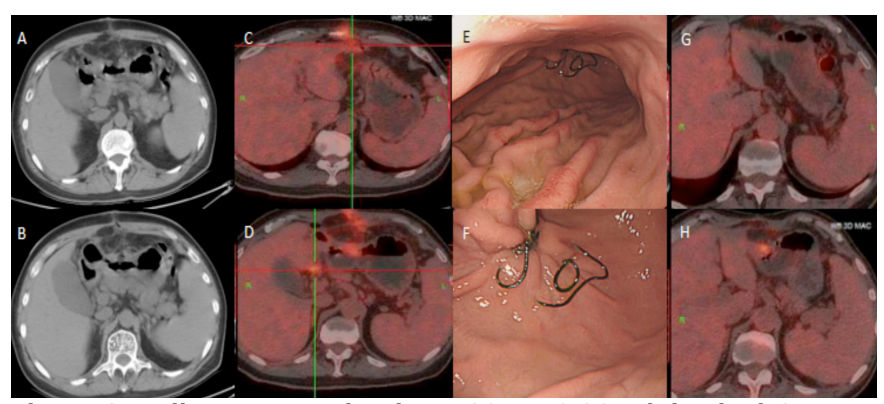

Figure 3: Follow-up examinations. 2017.10.20, abdominal CT scan showed local thickening of the antrum(A, B); 2018.5.7, PET-CT showed mild localized thickening of the antrum anterior wall, increased uptake of FDG drugs, SUVmax was 5.1, glycometabolism increased(C, D); 2018.5.8, gastroscopy showed that the shape of the stomach was normal, sutures were visible on the anterior sinus wall of the gastric body, with smooth surface and good peristalsis ( $E, F$ ); 2019.2.15, PET-CT showed no obvious thickening of gastric wall and abnormal uptake of FDG drugs; compared with PET/CT on May 7, 2018, the high metabolic lesions in the original gastric antrum disappeared (G, H). (Abbreviations: PET-CT positron emission tomography-computed tomography).

A decision of damage control surgical protocol was considered as better treatment strategy. Thus, the patient was subjected to gastric perforation repair and gastric mass biopsy. Postoperative pathological examination showed diffuse infiltration of atypical lymphoid cells in the stomach 
wall (Figure 2A, B). Immunohistochemistry staining showed CD20 (+), CD79a (+), BCL-6 (+), MuM-1 (-), CD10 (-), BCL-2 $(-)$, Cyclin D1 (-), CD38 (-), CD117 (-), Dog-1 (-), CD34 (-), Vimentin (-), S-100 (-), SMA (-), Desmin (-), CK (-), CK8/18 (-), Villin (-), Synaptophysin (-), STAT6 (-), TLE1 (-), and Ki-67 (+, $80 \%$ )(Figure 2C, D, E, F, G, H). The patient was finally diagnosed with gastric non-Hodgkin's diffuse large B-cell lymphoma (DLBCL), of germinal center origin.

The patient was transferred to the hematology-oncology department after the postsurgical condition improved, and he received $\mathrm{R}-\mathrm{CHOP}$ regimen chemotherapy (Figure 3 ). The patient achieved good clinical outcomes and he is still alive till now.

\section{DISCUSSION}

Acute gastric perforation is one of the most common abdominal emergencies that is characterised by sudden onset, complex clinical condition and rapid progression. ${ }^{5}$

It could easily cause abdominal infection, electrolyte imbalance, internal milieu disorder, liver and kidney failure and coagulation disorder, and in severe cases, can even lead to death. When a patient has malignant tumor with perforation, it is even more difficult to make surgical plans. ${ }^{6}$

The concept of damage control surgery was initially established for patients with complex and severe trauma during war times, and in peace time, it is important salvage technique for the treatment of severely ill patients. The usage of standard and radical surgery for secondary diffuse peritonitis depends on the severity, duration, age and complications of abdominal sepsis. ${ }^{7}$ A study of 455 patients showed that after the physiological recovery, brief laparotomy and subsequent surgery to delay the final repair are effective clinical strategies for critically ill patients. Thus, extending the concept of damage control surgery from severe trauma to the secondary diffuse peritonitis and secondary intraperitoneal sepsis caused by gastrointestinal perforation is logical and has been widely accepted by surgeons globally.

In this study, we report a patient with gastric lymphoma and acute gastric perforation, which was quite difficult to diagnose and treat, especially in making surgical plan. Damage control surgery has been widely accepted nowadays which has proved to significantly improve the survival of critically ill patients. When admitted into our hospital, the patient's condition was deteriorating with severe infection, abnormal hemodynamics and deranged coagulation parameters. Given that the patient was in critical condition and could not tolerate radical gastrectomy, we planned, according to damage control surgery, gastric perforation repair and gastric tumor biopsy, which we thought were most appropriate steps to reduce trauma and shorten operation time. There was no opportunity to complete full set of examinations and improve his general condition for surgical toler- ance. The intraoperative exploration confirmed gastric tumor with perforation, but further pathological diagnosis was needed. Herein, damage control surgery was helpful to avoid unreasonable radical gastrectomy and postoperative complications, and more importantly, to win the time for patients to recover and obtain pathological diagnosis. Finally, the patient achieved good clinical outcomes after 4 cycles of RCHOP regimen chemotherapy (Figure 3G, H). Our report provides insight into the value of damage control surgery in avoiding misdiagnosis and mistreatment.

\section{PATIENT'S CONSENT:}

Informed consent was obtained from the patient for publication of this case report and accompanying data.

\section{CONFLICT OF INTEREST:}

The authors declared no conflict of interest.

\section{AUTHORS' CONTRIBUTION:}

GC: Conceived and designed the study, acquisition of the data; drafted and revised the work; performed the operation; finally approved version to be published; agreed to be accountable for all aspects of the work.

MZ: Postoperative follow-up, analysed the data; drafted and revised the work; finally approved of the version to be published; agreed to be accountable for all aspects of the work.

JZ: Performed the operation, drafted and revised the work; approved the version to be published; agreed to be accountable for all aspects of the work.

JM: Performed the operation; postoperative follow-up, analysed the data; approved the version to be published; agreed to be accountable for all aspects of the work.

YL: Performed the operation, conceived and designed the work; revised the work; approved the version to be published; agreed to be accountable for all aspects of the work.

\section{REFERENCES}

1. Stone HH, Strom PR, Mullins R. Management of the major coagulopathy with onset during laparotomy. J Ann Surg 1983; 197(5):532-5. doi: 10.1097/00000658-19830500000005.

2. Rotondo MF, Schwab CW, McGoniga MD, Phillips GR, Fruchterman TM, Kauder DR, et al. Damage control: An approach for improved survival in exsanguinating penetrating abdominal injury. J Trauma 1993; 35(3):375-82.

3. Cheong JY, Keshava A. Management of colorectal trauma: A review. ANZ J Surg 2017; 87(8):547-53. doi: 10.1111/anS.13908.

4. Girard E, Abba J, Boussat B, Trilling B, Mancini A, Bouzat P, et al. Damage control surgery for non-traumatic abdominal emergencies. World J Surg 2018; 42:965-73. doi: 10.1007/ s00268-017-4262-6.

5. Simonetti I, Puglia M, Tarotto L, Palumbo F, Esposito $F$, Sciuto $A$, et al. When traditions become dangerous: Intestinal perforation from unusual foreign body: Case 
report and short literature review. Eur J Radiol Open 2019; 6:152-5. doi: 10.1016/j.ejro.2019.04.002.

6. Kamiyama K, Sawada S, Fukuda T, Yamaguchi T, Kato $\mathrm{N}$, Kojima A, et al. A case of long-term survival following two-stage surgery for perforated advanced gastric cancer caused by chemotherapy. Gan To Kagaku Ryoho 2019; 46(4):701-4.
7. Güsgen C, Schwab R, Willms A. Therapy concepts for diffuse peritonitis: When laparoscopic lavage and when open abdomen. Chirurg 2016; 87(1):34-9. doi: 10.1007/ s00104-015-0104-y.

8. Weber DG, Bendinelli C, Balogh ZJ. Damage control surgery for abdominal emergencies. BrJ Surg 2014; 101(1): e109-18. doi: 10.1002/bjs.9360. 